Journal of Forecasting

J. Forecast. 28, 405-425 (2009)

Published online 6 November 2008 in Wiley InterScience

(www.interscience.wiley.com) DOI: 10.1002/for.1117

\title{
Forecasting Growth and Inflation in an Enlarged Euro Area
}

\author{
THOMAS FLAVIN, ${ }^{1}$ EKATERINI PANOPOULOU ${ }^{2}$ AND \\ THEOLOGOS PANTELIDIS ${ }^{1,3}$ \\ ${ }^{1}$ Department of Economics, Finance and Accounting, NUI \\ Maynooth, Ireland \\ ${ }^{2}$ Department of Statistics and Insurance Science, University of \\ Piraeus, Greece \\ ${ }^{3}$ Department of Economics, University of Crete, Greece
}

\begin{abstract}
We compare models for forecasting growth and inflation in the enlarged euro area. Forecasts are built from univariate autoregressive and single-equation models. The analysis is undertaken for both individual countries and EU aggregate variables. Aggregate forecasts are constructed by both employing aggregate variables and by aggregating country-specific forecasts. Using financial variables for country-specific forecasts tends to add little to the predictive ability of a simple AR model. However, they do help to predict EU aggregates. Furthermore, forecasts from pooling individual country models usually outperform those of the aggregate itself, particularly for the EU25 grouping. This is particularly interesting from the perspective of the European Central Bank, who require forecasts of economic activity and inflation to formulate appropriate economic policy across the enlarged group. Copyright (C) 2008 John Wiley \& Sons, Ltd.
\end{abstract}

KEY WORDS forecasting; financial variables; growth; inflation; aggregation

\section{INTRODUCTION}

Forecasting macroeconomic variables across a large number of diverse countries is a difficult task but one that is very much a reality for the European Central Bank (ECB) as it strives to formulate appropriate monetary policy for the enlarged euro area. Marcellino et al. (2003) have examined this issue with relation to the original 11 members $^{1}$ of the common currency group. However, with the accession of 10 new member states in 2004, the group has become even more diverse and hence forecasting economic variables becomes even more hazardous. For this reason, we again examine the issue of the best method of forecasting economic activity and inflation in the individual member states as well as at the euro zone aggregate level.

* Correspondence to: Thomas Flavin, Department of Economics, Finance and Accounting, NUI Maynooth, Maynooth, Co. Kildare, Ireland. E-mail: thomas.flavin@ nuim.ie

${ }^{1}$ Greece was excluded from their analysis.

Copyright (c) 2008 John Wiley \& Sons, Ltd. 
We concentrate on single-equation linear models. Swanson and White (1997) show that linear models outperform non-linear alternatives for US forecasts of economic activity and inflation. Banerjee and Marcellino (2006) conclude that simple forecasting mechanisms work best and note the relatively good performance of the pure autoregressive model. Furthermore, these relatively simple models have often been found to outperform multivariate models in their out-of-sample forecast accuracy, especially in periods of economic change (see Marcellino et al., 2003). Given the recent period of economic and institutional change in European Union (EU) countries, especially the 10 new members, we favour the relatively low-parameterized single equation models. In particular, we examine a range of nested models using the simple autoregressive model as a benchmark and augmenting it with a number of other economic and financial variables which the extant literature has shown to be potentially useful in economic forecasting. We initially assess their forecasting ability by analysing their mean squared forecast errors (MSFEs). We extend this approach by testing for statistical differences in forecast accuracy, using the test statistic suggested by McCracken (2007). This test gives us a clear comparison of competing models and provides an advance on other studies of economic forecasting within the enlarged EU.

Having settled on the simple autoregressive model as a benchmark, our task is to choose a set of financial variables to include as predictors in alternative specifications. ${ }^{2}$ A voluminous literature exists on the choice of candidate variables but there is little consensus as to what the appropriate variables should be. In particular, some variables do well in some periods but their performance does not seem to be robust across time or indeed across countries. ${ }^{3}$ We choose a set of variables that are ubiquitous in the literature. We include forward-looking financial variables-stock market returns, short-term interest rates and the dollar exchange rate- that are thought to embody future economic expectations. In the case of output growth, studies such as Barro (1990), Fama (1990), Lee (1992), Estrella and Mishkin (1998), Hassapis and Kalyvitis (2002), Hassapis (2003) and Panopoulou et al. (2005), among others, find that stock market returns improve forecasting ability. Stock market returns are not generally found to be useful in predicting future inflation (e.g., Goodhart and Hoffman, 2000a). Interest rate measures have also enjoyed success in predicting output growth. Both shortterm rates are used (see Bernanke and Blinder, 1992) and, more usually, term spreads (see Harvey, 1988; Stock and Watson, 1989; Davis and Fagan, 1997). ${ }^{4}$ These have mixed forecasting performance and there is evidence that in the USA their ability to predict output growth has fallen over the past two decades (e.g., Haubrich and Dombrosky, 1996). For our inflation forecasts, we include the dollar exchange rate as a predictor. This is a potentially important channel through which inflation can be imported and has also been shown to be a useful predictor by Goodhart and Hoffman (2000b) for a range of countries. Stock and Watson (1999) find little evidence that exchange rates help the precision of output growth forecasts. We also investigate the forecast performance of the domestic money supply as well as extraneous influences in the form of US aggregates of the variable to be forecast. Money supply growth has been used in both output and inflation forecasting exercises by Stock and Watson (2003), while the effect of US variables on their EU equivalents has been documented by Marcellino et al. (2003).

We find that in the vast majority of cases financial variables add little predictive content over and above that already contained in the autoregressive model. US variables are only useful at the 1-month

\footnotetext{
${ }^{2}$ For the USA, Ang et al. (2007) show that the surveys outperform macro and asset market variables in predicting inflation. However, such surveys are not available for the enlarged euro area.

${ }^{3}$ For an excellent review of the literature, see Stock and Watson (2003).

${ }^{4}$ We use short-term rates as long yields are not available for many of the accession countries over our sample period.
} 
horizon, while other variables offer improvements at some longer horizons. However, consistent with the extant literature, it is difficult to identify any useful patterns that would help the researcher to forecast at the country level. For those forecasting aggregate variables our results have stronger implications. We find that pooling forecasts from individual country models (using a GDP weighted average) is consistently better than directly forecasting from the aggregate variable. This always holds for output growth and for the EU25 group in the case of inflation.

Our paper is organized as follows. The next section presents our econometric methodology. The third section describes the data and presents our empirical findings, while the fourth section offers some policy implications. The fifth section summarizes the main findings of the paper.

\section{ECONOMETRIC METHODOLOGY}

In this section, we briefly review the forecasting methodology employed to evaluate the forecasting accuracy of various models in a parametric setup for output growth and inflation in the 25 countries of the enlarged euro area. Although similar to that of previous studies (see, for example, Marcellino et al., 2003; Stock and Watson, 2003), we implement a different procedure to generate the out-ofsample forecasts. More specifically, our forecasting exercise is organized so that our benchmark model is always nested within the other estimated models. Thus, in contrast to other studies such as Stock and Watson (2004) and Favero and Marcellino (2005), we are able to perform formal statistical tests to compare the relative forecasting performance of alternative models.

We estimate several univariate models for each series to be forecast and focus on forecast horizons, $h$, of 1-, 3- and 12-month periods. ${ }^{5}$ In general, there are two alternative methods to generate multiperiod-ahead forecasts of a series. Specifically, the multiperiod-ahead forecast is constructed by iterating forward a one-period-ahead model or alternatively by estimating a horizon-specific model that can provide direct multiperiod-ahead forecasts. Asymptotic theory suggests that if the one-period ahead model is correctly specified, the MSFE of the iterated forecasts is lower than that of the direct forecasts (see Ing, 2003). However, if the models are misspecified, asymptotic theory suggests that the direct forecasts are more accurate than the iterated forecasts (in terms of the MSFE criterion). In this study, we generate forecasts for the variables of interest based on simple univariate models that are most likely approximations of the true data-generating mechanism. Therefore, we choose to implement the direct forecasting methodology based on the following horizon-specific model:

$$
y_{t+h}^{h}=c+a(L) y_{t}+B(L)^{\prime} Z_{t}+\varepsilon_{t+h}^{h}
$$

where $c$ is a constant, $a(L)$ is a scalar lag polynomial, $B(L)$ is a vector lag polynomial, $Z_{t}$ is a vector of financial (predictor) variables and $y_{t+h}^{h}=\sum_{s=t+1}^{t+h} y_{s}$. In our analysis, $y_{t+h}^{h}$ represents the growth of output and consumer prices over the next $h$ periods. ${ }^{6}$ Our specification of $Z_{t}$ differentiates the models. The number of lags for both $y_{t}$ and $Z_{t}$ is selected by the Schwartz Bayesian information criterion (SIC), setting the maximum lag length at 12 to avoid estimating models with low degrees of freedom.

\footnotetext{
${ }^{5} \mathrm{We}$ also forecast at the 6-month horizon but the pattern of results is similar to other horizons and hence are omitted for brevity.

${ }^{6}$ The $h$-step-ahead projection approach has an important advantage over the traditional one, in that no additional equations need to be estimated in order to simultaneously forecast the remaining variables of the model at hand.
} 
Setting $B(L)$ equal to zero provides us with the simple autoregressive model (AR), which will be used as a benchmark when evaluating our various forecasts. We estimate a number of alternative models by changing the composition of $Z_{t}$ for each of the 25 countries. As already mentioned, the estimation procedure is designed to allow us to implement formal statistical tests for the comparison of the MSFEs of the alternative models. More specifically, we first estimate an AR model for each country by setting $B(L)=0$. Out-of-sample forecasts are generated recursively. In each step, the AR model is re-estimated by keeping the lag order fixed, providing us with a sequence of forecasts. We then estimate alternative models by adding $Z_{t}$ to our model. We keep the order of $a(L)$ fixed $^{7}$ and once more use the SIC to select the order of $B(L)$. Consequently, the AR is always nested within the alternative models.

In addition to the individual countries, the preceding methodology is applied to three aggregated series (EU12, EU15 and EU25). ${ }^{8}$ The relevant aggregated series are constructed as the weighted average of the country-level data for all countries. A fixed-weighting scheme is employed using each country's GDP share in the euro area aggregate in PPP exchange rates averaged over $2005 .{ }^{9}$

For each of the 28 series ( 25 countries plus three aggregates), the forecasting performance of the various models is assessed by comparing the MSFE of the more richly specified model to that of the benchmark AR model. A lower MSFE suggests potential superiority of the candidate model over the AR model and may indicate that the candidate financial variable(s) is (are) a useful predictor for the variable of interest (i.e., output growth or inflation). However, it does not necessarily imply that the alternative model generates better forecasts than the benchmark. The lower MSFE may be due to sample variation. In order to establish superior forecast precision, one has to apply formal statistical tests. We use the following $F$-statistic proposed by McCracken (2007) to compare the forecasting performance of nested models:

$$
\text { OOS }-F=\frac{\sum_{t=1}^{P}\left[\varepsilon_{1, t}^{2}-\varepsilon_{2, t}^{2}\right]}{P^{-1} \sum_{t=1}^{P} \varepsilon_{2, t}^{2}}
$$

where $\varepsilon_{i, t}, i=1,2$ are the forecast errors of the restricted (AR) and the alternative unrestricted model, respectively, and $P$ is the number of out-of-sample forecasts. Under the null hypothesis, the two models have equal MSFE, while under the alternative the MSFE of the unrestricted model is less than that of the restricted. Thus, it is a one-sided test. The limiting distribution of the test statistic is non-standard but pivotal and numerical estimates of the asymptotic critical values for valid inference are provided by McCracken (2007). This statistic can be used for one-step-ahead forecasts.

We use four different variables to forecast output growth and inflation. For each country and aggregate series, $Z_{t}$ in the estimated models contains one of the four available predictors or all four predictors together. In the former case, the estimated model is nested within the 'general' model that contains all predictors. Thus, we can implement the test statistic, OOS- $F$, to compare the relative forecasting performance of the general model to that of models containing only one predictor.

\footnotetext{
${ }^{7}$ The lag-order of AR is allowed to be different across countries.

${ }^{8}$ EU12 corresponds to the 12 countries of the euro zone: Austria, Belgium, Finland, France, Germany, Greece, Ireland, Italy, Luxembourg, Netherlands, Portugal and Spain. EU15 represents EU12 plus Denmark, Sweden and the UK. Finally, EU25 augments EU15 with 10 new members: Cyprus, Czech Republic, Estonia, Hungary, Latvia, Lithuania, Malta, Poland, Slovakia and Slovenia.

${ }^{9}$ Source: Eurostat.
} 
In addition to forecasting the euro area aggregates directly using the respective aggregated series, we also consider pooling country-specific forecasts in order to construct the euro area forecast. The pooled forecasts can be constructed in different ways by varying the weighting scheme used. We consider two alternatives: (i) a fixed-weighting scheme based on each country's GDP share; and (ii) a simple equally weighted average of the country-specific forecasts. Although the first methodology seems more suitable, for comparison we apply both approaches. Asymptotic theory suggests that the pooled forecasts will be more accurate than those based on the aggregated series if the countryspecific models are time invariant, correctly specified and parameters differ across countries (See Lutkepohl, 1987). Finally, we should note that the test statistic described above is not valid for the comparison of the MSFEs produced by the forecasts of the aggregated series to those of the pooled forecasts.

\section{EMPIRICAL RESULTS}

In this section we report and discuss the results of applying the techniques outlined in the previous section to examine the empirical relationship between growth, inflation and financial variables in the euro area.

\section{Data}

We focus on forecasting inflation and output growth. Inflation is measured as the growth in consumer prices. We follow Ang et al. (2007) in predicting the level rather than the change in inflation. They put forward a number of reasons for assuming that inflation is a stationary process. We perform unit root tests on all inflation variables to confirm the stationarity of the series. ${ }^{10}$ To measure the growth rate of output, we use the industrial production index, which we transform in first logarithmic differences. Some US studies prefer the use of coincident indicators rather than industrial production as a proxy for economic growth on the basis that the latter is a shrinking share of the economy. However, for the countries in our analysis, such indicators are not available. Hence, we work with industrial production given that a consistent measure is available across all EU25 countries.

The predictive variables considered are short-term interest rates, stock market returns, money supply growth, exchange rates against the US dollar, US growth, US inflation, and domestic growth and inflation for the 25 countries. Our dataset is monthly and covers the period from January 1995 to April 2006. ${ }^{11}$ Many policymakers may prefer data measured at quarterly horizons but our choice of data frequency is driven by the need to have sufficient observations to produce meaningful econometric estimates and conduct an out-of-sample forecasting exercise. Our data sources are drawn from various sources and details are given in the Appendix. Money supply and stock market indices were seasonally adjusted where necessary. Interest rates and exchange rates are rendered stationary by employing the first difference and the first logarithmic difference, respectively. The fixed weights used for pooling forecasts are based on each country's GDP share in the euro area in PPP terms for 2005.

\footnotetext{
${ }^{10}$ Results are available upon request.

${ }^{11}$ To ensure homogeneity of our results, we employ the longest dataset for which data were available for the 25 euro area countries.
} 


\section{Models and forecast evaluation}

Our simulated out-of-sample forecasting experiment is conducted using a recursive methodology. The out-of-sample forecast period is 2003:05 to 2006:04 (36 observations), covering the recent period of monetary union and generating a ratio of out-of-sample $(P)$ over in-sample observations $(R)$ equal to approximately 0.36 . In each step, we re-estimate all the candidate models by adding one observation at a time. The $h$-step-ahead forecasts are generated for the periods of 1,3 and 12 months and the corresponding MSFEs are calculated. In an effort to conserve space, our forecasting analysis is restricted to the within-country and aggregate euro area forecasting ability of candidate variables. Including cross-country influences in this parametric setup would hugely increase the number of models and may obscure our main findings.

The models estimated in the forecasting experiment are as follows:

- Model (1): the benchmark AR model, i.e. $Z_{t}$ is excluded from (1).

- Models (2)-(5): for output growth, the AR model is augmented with lags of either stock market returns, interest rate changes, money supply growth or US growth. For inflation, the candidate variables are output growth, exchange rate returns, money supply growth and US inflation. In each specification, $Z_{t}$ contains only one predictor variable.

- Model (6): all of the candidate variables are added to the AR specification simultaneously.

All models were estimated for the 25 countries and the three euro area aggregates.

\section{Growth forecasts}

1-step ahead forecasts

Table I reports the MSFEs generated by models 1-6 for each country and aggregated series at the 1-month forecast horizon. Columns labelled EU12, EU15 and EU25 refer to results for aggregate series, while pooled forecasts are generated using both the GDP-weighted average (Pooled1) and the equally weighted average approach (Pooled2).

Focusing on our benchmark AR model, we find huge differences in forecasting performance across countries. From the original members of the single-currency area, relatively small MSFEs are recorded for Italy, Germany and Spain. On the other hand, this model produces large MSFEs for Luxembourg, Finland and Portugal, but it fails spectacularly to predict output growth in Ireland. Forecast errors of similar magnitude are recorded for Denmark, Sweden and the UK, while MSFEs for the accession countries tend to be larger, though the dispersion of values is again large, ranging from low values in Malta and Hungary to very high errors in Latvia and Lithuania. Therefore the AR model has mixed success in predicting future output growth across countries. This initial analysis shows the difficulty in forecasting economic activity across the enlarged EU. Therefore it is worth looking at the aggregate output growth variable. It is noteworthy that the forecast accuracy of the aggregate variable is consistently inferior to the pooled forecasts when the GDP weighted average is applied. This finding is similar to that of Marcellino et al. (2003) for their restricted group of EU countries. In contrast, pooled forecasts based on a simple average fare worst of all. This is due to over-weighting the smaller countries, such as Ireland, Latvia and Lithuania, where forecast performance is the poorest. Hendry and Clements (2004) propose a number of reasons as to why pooled forecasts might out-perform the aggregate. They argue that pooling forecasts from various candidate models allows alternative models to act as 'intercept corrections', which have been shown to improve forecasts in the presence of structural breaks and/or model misspecification. They interpret crosscountry forecast combinations, as we employ here, as a specific type of 'intercept correction'. Finally, 


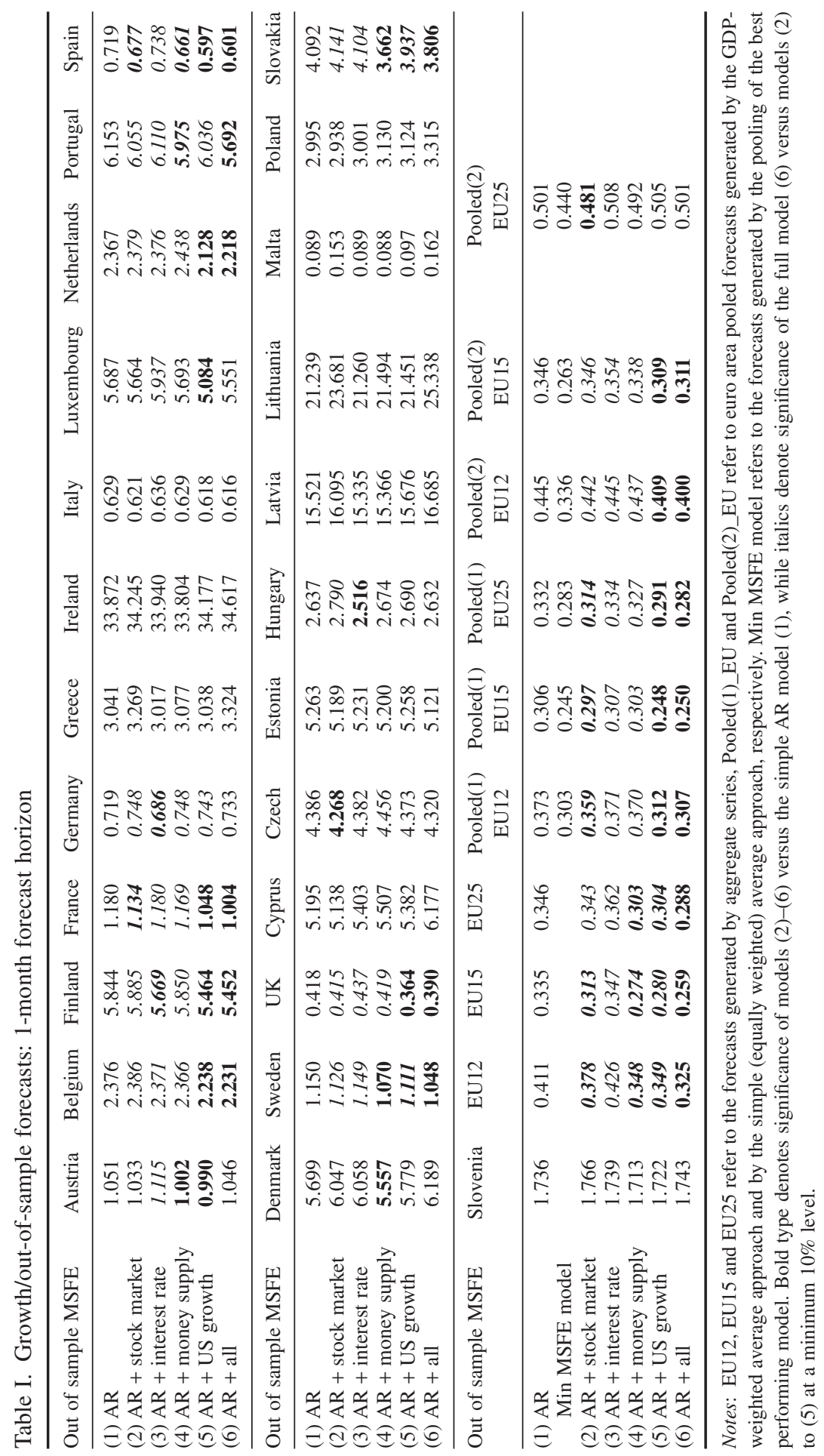


the superiority of the GDP-weighted pooled forecasts over the forecasts generated from the aggregated series also holds in regards to the minimum MSFE model.

The MSFE of the alternative models exhibit a similar pattern and we now compare the magnitude of these errors to the benchmark AR model. A lower MSFE implies that the added variable has predictive power over and above that contained in the lagged dependent variables. Improvements in forecast accuracy are observed for models that include the stock market as an additional predictor in $48 \%$ of the analysed countries, $40 \%$ for the short-term interest rate, $52 \%$ for the money supply, $64 \%$ for US growth and $60 \%$ for the model that includes all candidate variables. These improvements are more common in the EU15 than in the accession countries. Admittedly, many of the MSFE reductions are very small and may not be statistically significant. An alternative interpretation is that the AR model does well vis-à-vis its competitors for the individual country forecasts. In contrast, for the aggregate and the pooled forecasts, the augmented models generally do better than the AR model in terms of forecast precision. The MSFEs are generally lower and often substantially so. Of course, all of these forecast errors are generated subject to estimation error and hence we should perform proper statistical testing in order to properly evaluate the significance of our results.

We use the OOS- $F$ statistic calculated from (2) to compare each of the alternative models to the AR specification. Under the null hypothesis, the MSFE of the AR model equals that of the alternative model. When the table entry appears in bold, it signifies rejection of the null hypothesis. ${ }^{12}$ This statistical test further supports the adoption of the AR model as the best predictor of output growth. Very few of its competitors manage to outperform it-models including the stock market variable in only $12 \%$ of cases, $12 \%$ for the short-term interest rate, $24 \%$ for the money supply, $40 \%$ for US growth and $36 \%$ for the all-inclusive model. In the vast majority of cases the AR model produces forecasts that are at least as accurate as the other models. This is particularly evident for the accession countries, where the competing models are superior in only $10 \%$ of all possible country/variable combinations. Furthermore, even in countries where the AR model generated poor forecasts, such as Ireland, the additional predictors fail to improve on forecast accuracy. At this forecast horizon, it is difficult to identify any variable that reliably predicts output growth. Of the reported candidates, US growth tends to be the best predictor, suggesting that US economic conditions tend to lead European growth. In line with other studies, financial variables fare poorly in enhancing the accuracy of output forecasts (see Stock and Watson, 2003, and references therein).

An issue arises with respect to the selection of appropriate critical values for comparing the forecasting accuracy of alternative models in the case of pooled forecasts. The critical values depend on the number of additional parameters estimated in the unrestricted model. We set the number of parameters in the pooled statistic equal to the highest number of additional estimated parameters among the country-specific models. The results indicate that pooling the forecasts of the countryspecific models that include US growth as a predictor generates statistically lower MSFEs than pooling the simple AR models. The same result holds for the models that include the stock market returns when the pooled forecasts are calculated based on the GDP weights. Interestingly, pooling models that include money supply generates lower MSFEs compared to pooling the AR models but the differences are not statistically significant. Conversely, pooling the forecasts generated by models augmented with the interest rate variable produces larger MSFEs compared to the benchmark case.

\footnotetext{
${ }^{12}$ Given that McCracken (2007) does not tabulate critical values for $P / R$ equal to 0.36 , we base our inference on the critical values for $P / R$ equal to 0.4 .
} 
We also employ the OOS- $F$ statistic to test whether the MSFE of the 'general' unrestricted model (model 6) is lower than the MSFE of the single-predictor models. Again, the MSFEs are equal under the null and where this hypothesis is rejected the entry is represented in italics. For the individual countries, there is little statistical difference between models. For groups of countries, such as Germany, Greece, Ireland and Italy, there is no statistical evidence that the more highly parameterized model performs better than its restricted counterparts. Hence, at the country level, one should not automatically assume that larger models are preferred. However, this is completely reversed for the aggregate variable, with consistent evidence that the most general model does better than almost all the constrained alternatives. The only case where the general model is not statistically superior to a restricted model is when the restricted model uses US growth as a predictor, suggesting that much of the predictive content is in the US variable.

In unreported results, we evaluate the forecast precision of pairs of alternative models using a modified version of the Diebold-Mariano (1995) statistic for comparing non-nested models. There is little evidence of statistically significant differences between pairs of competing models. Furthermore, there is little pattern that would suggest which, if any, of the additional predictors should be chosen in the forecasting exercise. ${ }^{13}$

\section{h-step ahead forecasts}

Tables II and III report the results for the longer forecast horizons. The tables have the same format as before. The MSFE of the AR model generally increases with the forecast horizon. The model performs qualitatively the same as at the 1-month horizon, with countries such as Germany enjoying relatively good forecasts and Ireland and Latvia constantly having huge errors.

Evidence of competing models outperforming the benchmark reduces also. The importance of US growth as a predictor disappears as the horizon increases and only does better than the AR model in $12 \%$ of cases at the 12-month horizon. In general, predictors perform worse as we go further into the future. At the 12-month horizon, lower MSFEs are only produced in $32 \%$ of cases with the inclusion of the stock market return as a forecast variable, $28 \%$ for the short interest rate, $36 \%$ for the money supply, $12 \%$ for US growth and $28 \%$ for the general model. The main exception is the short-term interest rate in predicting future output growth in the 10 accession countries. At both horizons it has approximately a 50\% success rate in beating the AR model. Interestingly, the short rate does quite well at the 3-month horizon, outperforming the benchmark in over $70 \%$ of cases and across all country subsets. Similar to the 1-month forecast horizon, the GDP-weighted pooled forecasts consistently produce lower MSFEs than the forecasts based on the aggregated series for all forecast horizons and all groups of countries (i.e., EU12, EU15 and EU25). This result holds for both the AR and the minimum MSFE model.

Once more we test whether there is statistical evidence of model superiority over the benchmark. Again, evidence in favour of the alternative model is weak and performance is not robust across countries or time horizons. At these longer horizons, US growth has no additional predictive value. In some cases there is evidence that certain variables do better in certain countries. For example, the model including stock market returns outperforms the benchmark in Germany and the Czech Republic at all forecast horizons beyond 1 month, while the money supply variable adds predictive content over and above the benchmark at the same horizons for both France and Slovenia. However, there

\footnotetext{
${ }^{13}$ For brevity, these results are not reported but full details of the test and the results are available in the working paper version of this study, available at http://economics.nuim.ie/research/workingpapers/documents/N1950808.pdf 


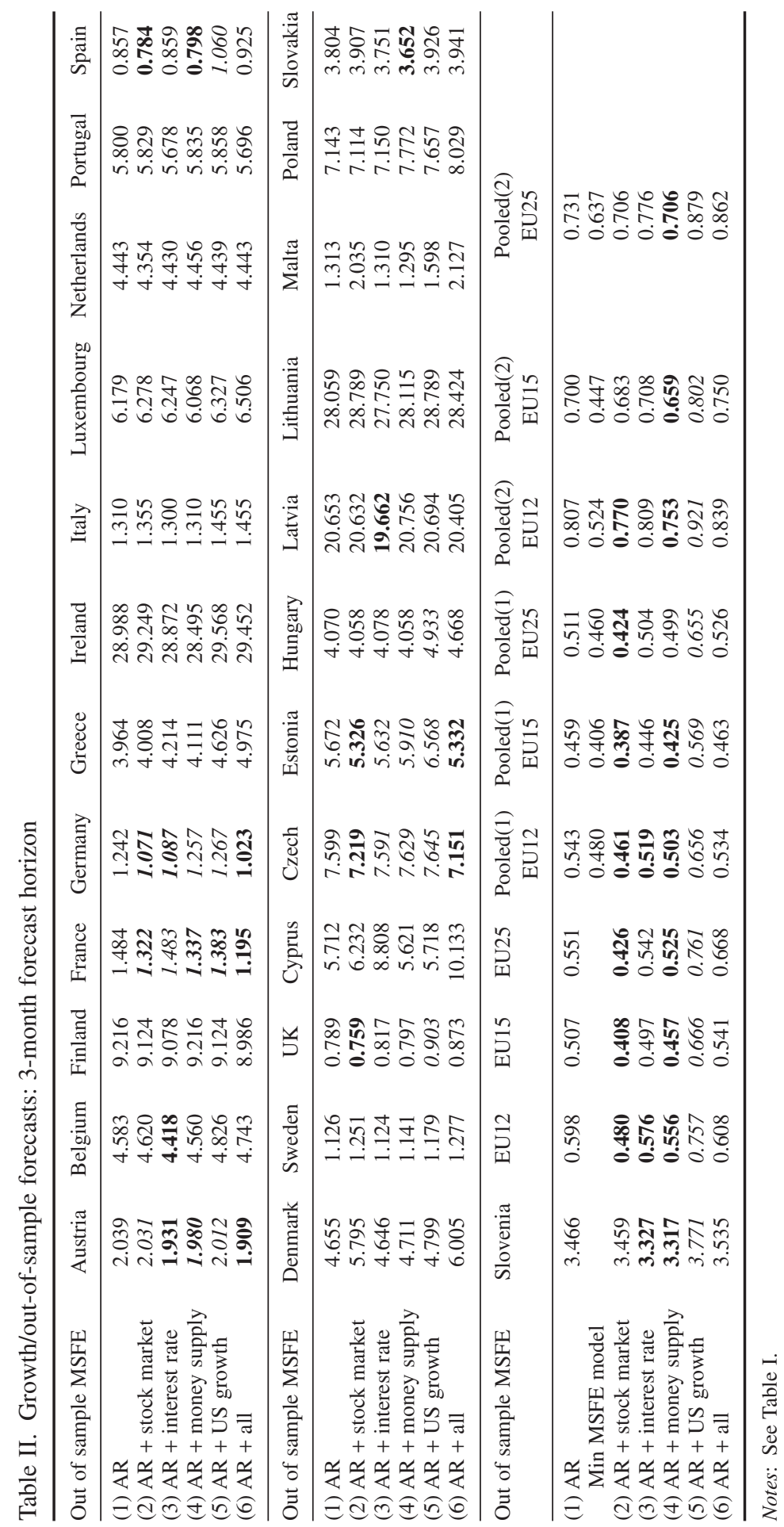

Copyright () 2008 John Wiley \& Sons, Ltd.

J. Forecast. 28, 405-425 (2009)

DOI: $10.1002 /$ for 


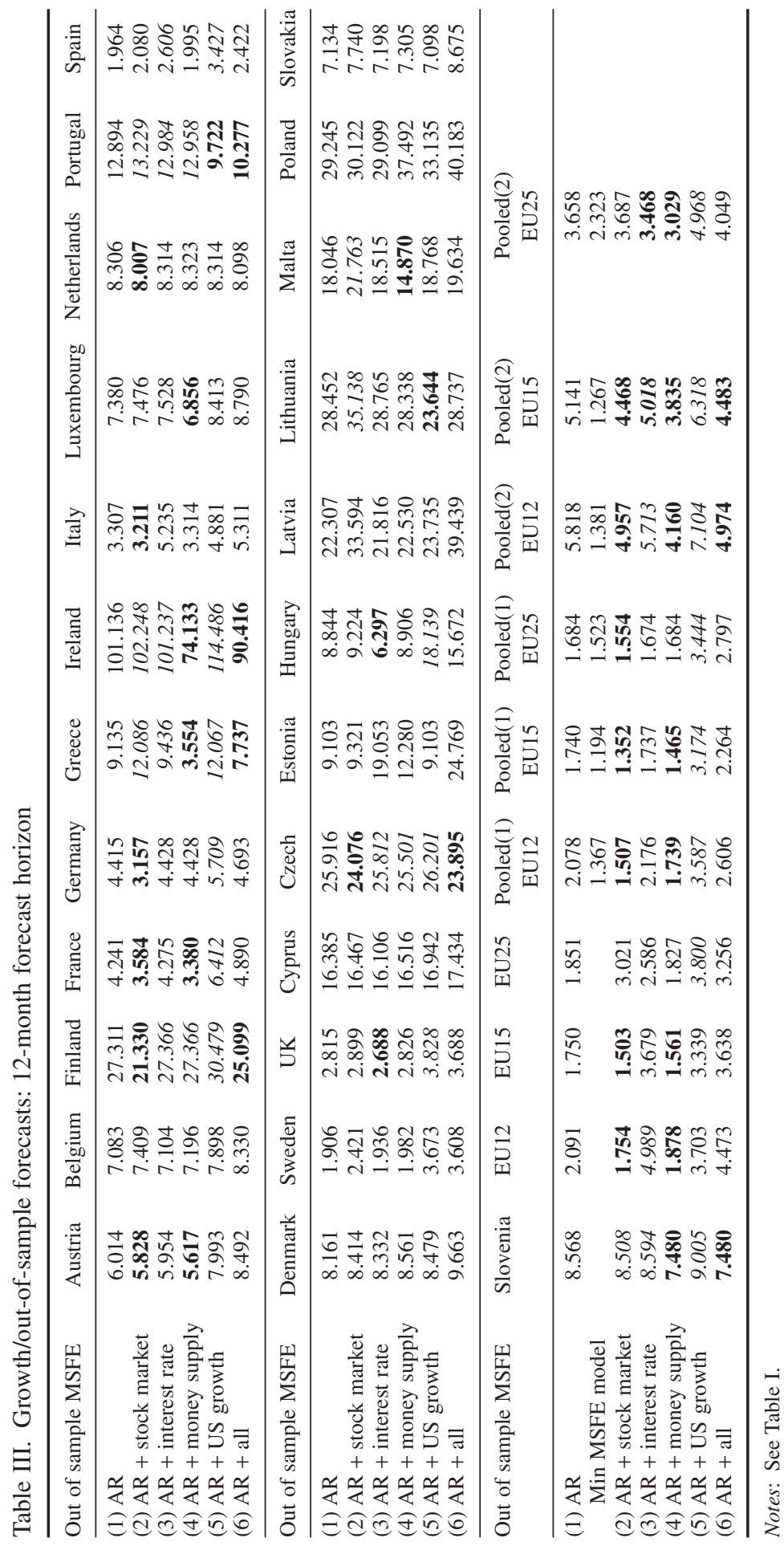

Copyright (c) 2008 John Wiley \& Sons, Ltd.

J. Forecast. 28, 405-425 (2009)

DOI: $10.1002 /$ for 
is no definite pattern in our results to help the researcher choose good predictors of output growth for individual countries or forecast horizons.

When comparing the aggregate variables, similar patterns are observed. Again the MSFE increases with the forecast horizon and, as before, pooling the forecasts using a GDP weighted average produces lower errors than forecasting with the aggregate itself. Pooling using the simple average does worst of all. Consistent with results for individual countries, the US growth rate has nothing to add to the benchmark, but all other variables produce lower MSFEs. Furthermore, if we focus on the GDP-weighted forecasts, models including the stock market return represent an improvement over the benchmark, and interestingly, this variable consistently adds predictive value at all forecast horizons and for all output growth aggregates. Moreover, pooling the forecasts of models that include money supply generates statistically significant lower MSFEs than pooling the benchmark model for both EU12 and EU15 (but not EU25) and for all forecast horizons greater than 1 month. Finally, contrary to the one-period horizon, the general model fails to outperform the restricted ones.

\section{Inflation forecasts}

\section{1-step-ahead forecasts}

Results for our 1-month inflation forecasts are contained in Table IV. The table follows the same format as for output growth.

Again, the simple AR model is our benchmark. A striking feature of our results is that, relative to output growth, the MSFEs are much smaller for inflation forecasts and there are no large outliers as in the previous analysis. On average, the AR model is more successful in predicting future inflation in the more traditional EU countries than in the accession countries, though it performs quite well for Poland and the Czech Republic. With regard to the aggregate variables, there is a marginal improvement from using pooled country forecasts rather than directly forecasting the aggregate. However, this is not as pronounced as in the case of output growth. It is noteworthy, however, that the largest reduction in MSFE is for the broadest and most diverse group, i.e., the EU25.

The predictive content of the economic and financial variables is initially assessed by comparing the magnitude of MSFEs. For our 1-month inflation forecasts, the candidate variables perform poorly relative to the pure AR model. Focusing on the individual countries, the AR model is at least as good as its competitors in the majority of country/variable combinations. Additional predictive ability is only present in $32 \%$ of cases with the inclusion of the economic growth variable, $32 \%$ for the dollar exchange rate, $28 \%$ for the money supply, $44 \%$ for US inflation and $32 \%$ for the general model. As before, at this horizon, the most successful variable in terms of enhancing the forecast accuracy of the AR model is the US counterpart. This predictor performs better for the EU12 group than the average, with a reduction in MSFE recorded in 58\% of countries. For the aggregate variables, augmenting the AR model with either economic growth or the exchange rate tends to reduce the forecast error for all country groups but poorer performance is associated with models including the money supply and US inflation.

Given that the reductions in MSFE recorded are often small, we test whether the differences are statistically significant. For the individual countries, none of the candidate variables consistently add predictive content to the AR model. In fact, with the exception of US inflation, evidence of statistically significant improvements are rare: one for the money supply and three for both economic growth and the dollar exchange rate. Augmenting the AR model with US inflation results in a statistical improvement in forecast accuracy in six countries (with four in the EU12). Therefore, at the country level, the AR model tends to be the dominant forecast model among our set of candidates. At the aggregate level a similar story emerges. For EU12 and EU15 aggregate variables, nothing 


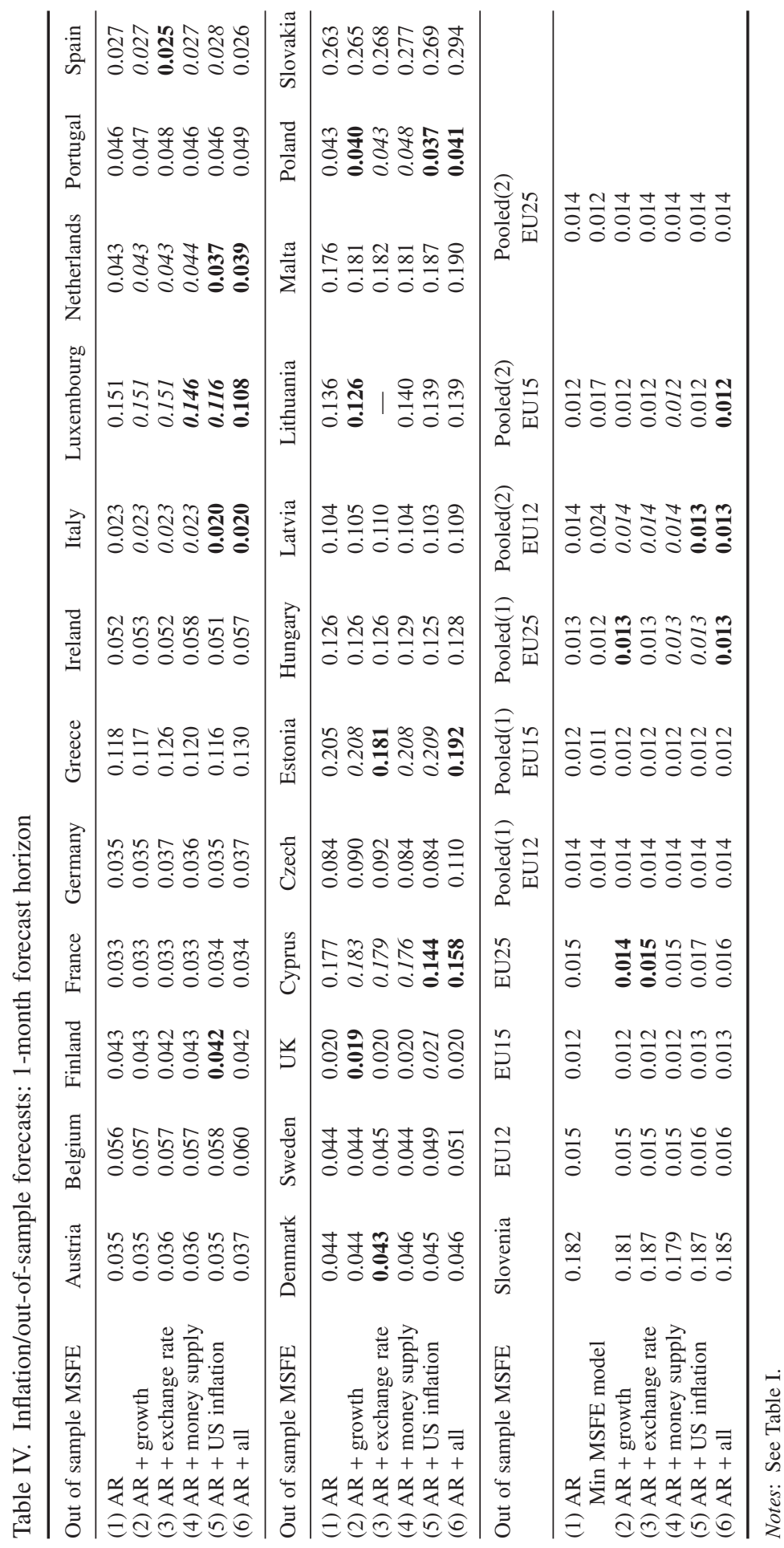

Copyright (c) 2008 John Wiley \& Sons, Ltd.

J. Forecast. 28, 405-425 (2009)

DOI: $10.1002 /$ for 
outperforms the AR forecast, while there is some evidence that the broadest EU25 aggregate is more accurately forecast by models including economic growth and the exchange rate. In regard to the pooled forecasts, competing models fail to outperform the simple AR in almost all cases. The EU25 aggregate is a noteworthy exception, where pooling the forecasts of models (weighted by GDP share) including output growth produces statistically significant lower MSFE than pooling the benchmark.

Evidence of the poor performance of the forecasting variables is compounded by testing for superiority of the general model, where we show that in the majority of cases there is no statistical support for the hypothesis that the general model does better than the less parameterized versions. For the majority of countries, the hypothesis of equal MSFEs cannot be rejected. However, this unrestricted model has some support in Luxembourg, Italy and the Netherlands. As for output growth, we compute the modified Diebold-Mariano statistic for pairwise comparison of alternative models but, again, no useful pattern emerges. Some models outperform others in certain countries but there is no systematic pattern to help researchers or policymakers identify the 'best' forecast model.

\section{h-step-ahead forecasts}

We next turn our attention to the accuracy of inflation forecasts at longer horizons, namely 3 and 12 months. Results are presented in Tables V and VI, and are discussed below with particular attention to the differences vis-à-vis the 1-month forecasts.

Once more our benchmark forecasts become less precise as we increase the time horizon. Generally the same pattern of accuracy is observed across time periods, with relatively small errors in France and Italy compared to those for Estonia and Slovakia. In contrast to the unambiguous result for output growth that GDP-weighted pooled forecasts deliver smaller MSFEs than forecasting the aggregate directly, this does not consistently hold for inflation forecasts. The only case where the pooled forecasts regularly outperform the forecasts based on the aggregated series for all forecast horizons is for the EU25. For the EU12 and EU15, at the 3- and 12-month horizons, the aggregate forecast records smaller errors than the pooled forecast. However, in most cases the difference in MSFE between the two methods is small.

Introducing our predictor variables, we find that instances of improved forecast accuracy, as measured by reduction in the MSFE, are uncommon and decline with time. However, there are some notable exceptions. The most striking is the dollar exchange rate at the 3-month horizon. Here it delivers a lower MSFE in $68 \%$ of the countries. It performs best in the EU12, with nine of 12 countries having a reduced MSFE. Even in the accession countries, the majority (six of 10) experience improved prediction. As for output growth, the US equivalent proves useful only at the 1-month horizon. Its additional value fades and has almost completely disappeared at the 12-month horizon. However, some variables have predictive content for certain countries and are robust across all time horizons beyond 1 month. The most prevalent of these is the exchange rate. This variable adds statistically significant forecast accuracy in Finland, Ireland, Denmark, Estonia and Poland. Money supply delivers similarly consistent gains in Cyprus and Luxembourg, while output growth generates more precise predictions in Poland. The unrestricted model outperforms the AR specification in Luxembourg, Denmark, Latvia and Slovenia.

For aggregate inflation forecasts, there is no single variable that consistently delivers error reduction vis-à-vis the benchmark, though the unrestricted model improves accuracy for the EU25 aggregate. At the 3-month horizon, the exchange rate has predictive value but this disappears at the longer horizon. For pooled forecasts, only two models manage to outperform the simple AR. First, for 


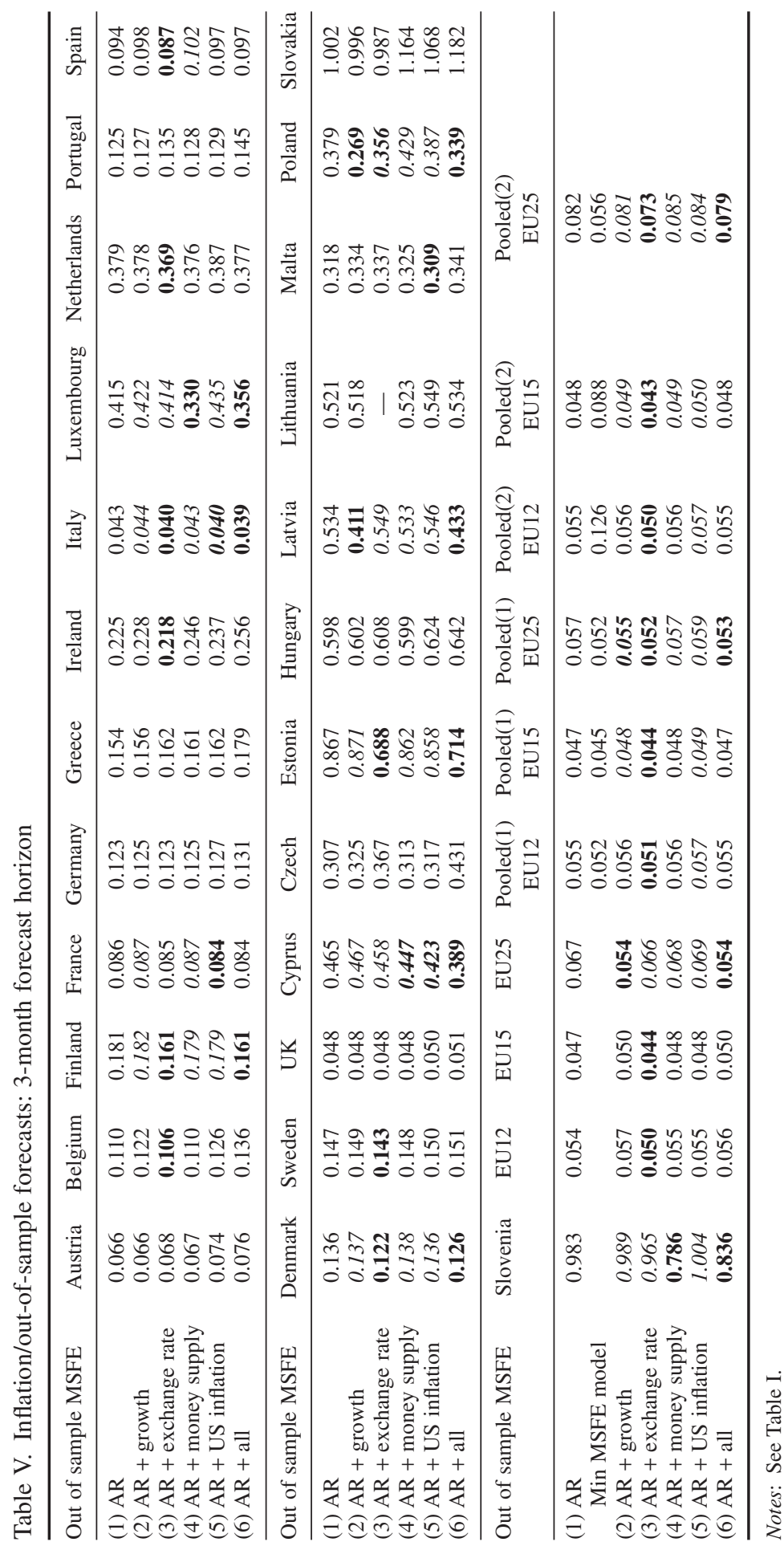

Copyright @ 2008 John Wiley \& Sons, Ltd.

J. Forecast. 28, 405-425 (2009)

DOI: $10.1002 /$ for 


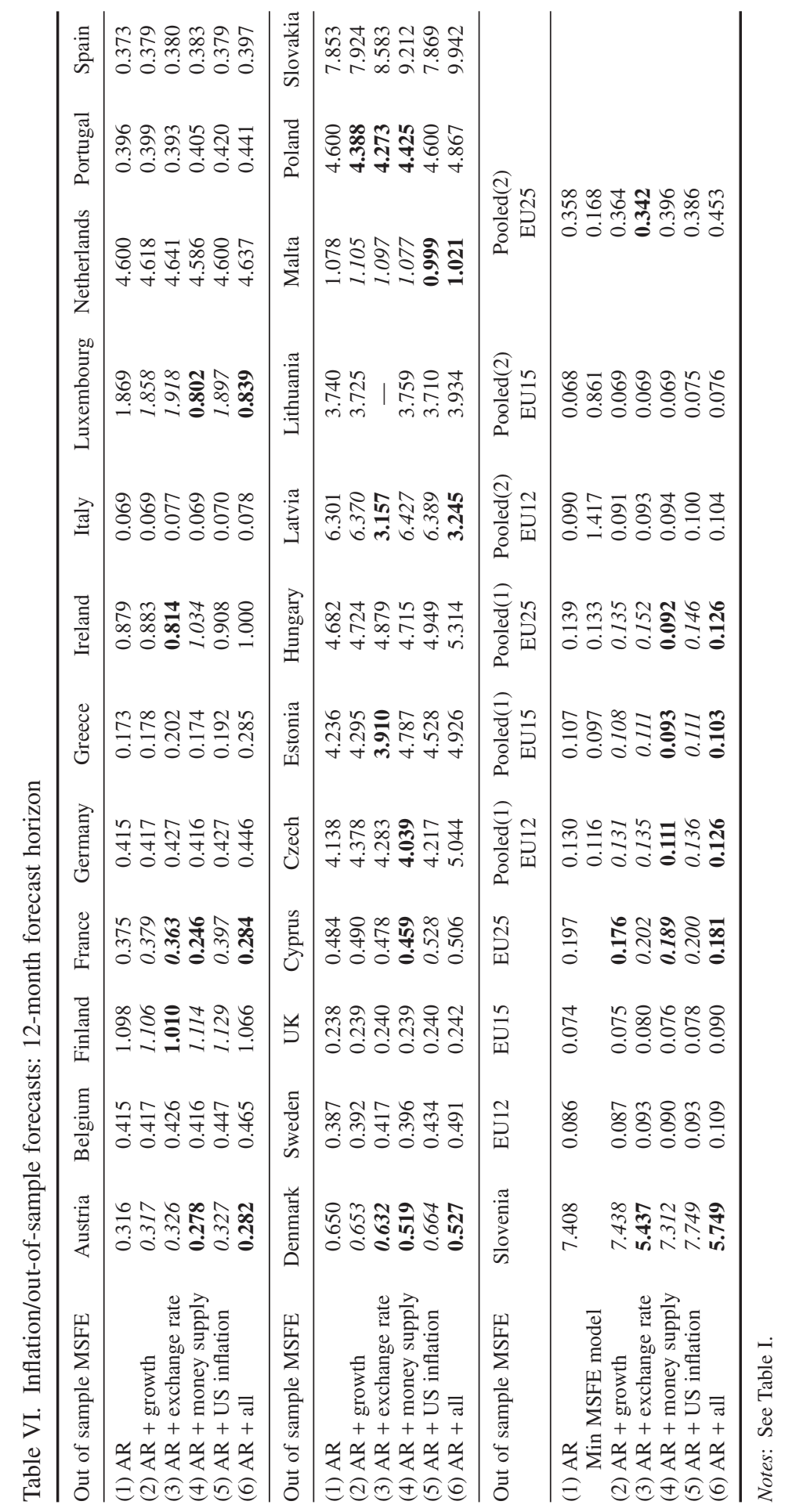

Copyright (@ 2008 John Wiley \& Sons, Ltd.

J. Forecast. 28, 405-425 (2009)

DOI: $10.1002 /$ for 
$h=12$, the weighted average of models including the money supply produces MSFEs that are statistically lower than those of the AR. Second, at the 3-month horizon a similar result holds for models that include the exchange rate. Finally, the general model consistently fails to outperform the restricted ones.

\section{Overview and in-sample results}

A number of important points emerge from our analysis. For forecasters of the aggregate variables, pooling forecasts of the individual countries models generally delivers better forecasts than directly forecasting from the aggregate. This is particularly evident for output growth. Table VII presents the MSFEs of both the AR and the best-performing alternative model. Across all country groupings and all forecast horizons, pooled forecasts of economic activity are more precise than those generated directly from the aggregate variable. These differences are quite large, especially for the best models at the longer horizons. Given the wide range of output growth rates across countries, pooling is important in that it allows the 'correction' to the benchmark not facilitated by the direct forecast. The picture for inflation forecasts is not as clear but, importantly, for the EU25 aggregate, pooled forecasts again outperform the aggregate at all time horizons. For the other country groupings, there is no clear pattern but at the shorter horizons the difference between the two forecasting methods is small.

At the country level, the forecasting performance of the predictor variables is poor. Most often the simple AR specification is not surpassed by more richly specified models. There are particular

Table VII. MSFE of the forecasts of the aggregate series

\begin{tabular}{lllllll}
\hline \multicolumn{3}{c}{ Output growth } & & Inflation \\
\hline AR model & & & & & & \\
$h=1$ & $E U 12$ & $E U 15$ & $E U 25$ & $E U 12$ & $E U 15$ & $E U 25$ \\
Pooled & $\mathbf{0 . 3 7 3}$ & $\mathbf{0 . 3 0 6}$ & $\mathbf{0 . 3 3 2}$ & $\mathbf{0 . 0 1 4}$ & $\mathbf{0 . 0 1 2}$ & $\mathbf{0 . 0 1 3}$ \\
Aggregated & 0.411 & 0.335 & 0.346 & 0.015 & 0.012 & 0.015 \\
$h=3$ & $E U 12$ & $E U 15$ & $E U 25$ & $E U 12$ & $E U 15$ & $E U 25$ \\
Pooled & $\mathbf{0 . 5 4 3}$ & $\mathbf{0 . 4 5 9}$ & $\mathbf{0 . 5 1 1}$ & 0.055 & 0.047 & $\mathbf{0 . 0 5 7}$ \\
Aggregated & 0.598 & 0.507 & 0.551 & $\mathbf{0 . 0 5 4}$ & $\mathbf{0 . 0 4 7}$ & 0.067 \\
$h=12$ & $E U 12$ & $E U 15$ & $E U 25$ & $E U 12$ & $E U 15$ & $E U 25$ \\
Pooled & $\mathbf{2 . 0 7 8}$ & $\mathbf{1 . 7 4 0}$ & $\mathbf{1 . 6 8 4}$ & 0.130 & 0.107 & $\mathbf{0 . 1 3 9}$ \\
Aggregated & 2.091 & 1.750 & 1.851 & $\mathbf{0 . 0 8 6}$ & $\mathbf{0 . 0 7 4}$ & 0.197 \\
Best model & & & & & & \\
$h=1$ & $E U 12$ & $E U 15$ & $E U 25$ & $E U 12$ & $E U 15$ & $E U 25$ \\
Pooled & $\mathbf{0 . 3 0 3}$ & $\mathbf{0 . 2 4 5}$ & $\mathbf{0 . 2 8 3}$ & $\mathbf{0 . 0 1 4}$ & $\mathbf{0 . 0 1 1}$ & $\mathbf{0 . 0 1 2}$ \\
Aggregated & 0.325 & 0.259 & 0.288 & 0.015 & 0.012 & 0.014 \\
$h=3$ & $E U 12$ & $E U 15$ & $E U 25$ & $E U 12$ & $E U 15$ & $E U 25$ \\
Pooled & $\mathbf{0 . 4 8 0}$ & $\mathbf{0 . 4 0 6}$ & 0.460 & 0.052 & 0.045 & $\mathbf{0 . 0 5 2}$ \\
Aggregated & 0.480 & 0.408 & $\mathbf{0 . 4 2 6}$ & $\mathbf{0 . 0 5 0}$ & $\mathbf{0 . 0 4 4}$ & 0.054 \\
$h=12$ & $E U 12$ & $E U 15$ & $E U 25$ & $E U 12$ & $E U 15$ & $E U 25$ \\
Pooled & $\mathbf{1 . 3 6 7}$ & $\mathbf{1 . 1 9 4}$ & $\mathbf{1 . 5 2 3}$ & 0.116 & 0.097 & $\mathbf{0 . 1 3 3}$ \\
Aggregated & 1.754 & 1.503 & 1.827 & $\mathbf{0 . 0 8 7}$ & $\mathbf{0 . 0 7 5}$ & 0.176 \\
\hline
\end{tabular}

Notes: MSFEs of GDP-weighted pooled forecasts. Bold type denotes the lower MSFE. Best model refers to the model with the minimum MSFE. 
time horizons and countries when additional variables deliver statistically significant forecast improvements but these are not persistent over time or across countries. While most candidate variables receive some statistical support at some horizon, no definite pattern emerges which would allow the researcher to be confident that a given variable will improve forecast accuracy across a broad range of countries and over different forecast horizons. This is consistent with Banerjee and Marcellino (2006), who find that leading indicators change over time so real time forecasts may be unreliable. Of course, this may be simply due to the fact that the variables that we have identified from the extant literature are just not suitable. To examine this possibility, we analyse their in-sample performance using likelihood ratio tests. For output growth, we note two features. Firstly, in-sample significance increases with the horizon; for example, US growth is only statistically significant in $28 \%$ of countries at the 1-month horizon, but for the 12-month forecast this proportion grows to $76 \%$. Secondly, in-sample significant relationships are more common for the older EU countries than the new accession states; for example, at the 3-month horizon money supply is significant for $20 \%$ of the EU15 as opposed to $0 \%$ for the new members. ${ }^{14}$ In general, in-sample predictability does not imply out-of-sample forecast accuracy. Similar to previous studies, variables that perform well within sample often fail to repeat this success out of sample. This is indicative of instability in the forecasting relationships which has been documented by Stock and Watson (2003) and is likely to be present in the new enlarged EU as macroeconomic policies become streamlined across countries and economic and financial institutions change in many member states-radically in some cases.

\section{POLICY IMPLICATIONS}

Our analysis has a number of policy implications. At the country level, simple models appear to do relatively well. Our benchmark AR model is as good as its competitors in the vast majority of cases. Forecasters should be wary of over-parameterizing forecasting equations because there is little evidence to suggest that in-sample predictability persists to the out-of-sample period. This is consistent with Stock and Watson (2003). In our analysis, forward-looking financial variables enjoy limited success at horizons beyond 1 month, e.g. stock market return and exchange rates, but one should be cautious about the stability of the relationship.

From an EU perspective, a more interesting question may be how to forecast aggregate variables across the new enlarged group. Here our results provide an interesting insight. For output growth our results are clear. As in Marcellino et al. (2003), for their sample of the original euro members, we find that pooling forecasts from individual country models is more accurate than directly forecasting the aggregate. The flexibility offered by this approach delivers more accurate predictions. Moreover, the larger the group, the bigger the gains in forecast error reduction. This is likely to be important with the accession of the new, highly diverse states. We have already noted that these country variables are most difficult to predict, so this result offers hope to the researcher that individual errors can be reduced. Furthermore, when combining forecasts we advocate the use of a GDP weighted average to reflect the contribution of each member state. Given that forecasts for larger countries tend to be more precise, this contributes to the relative accuracy of the prediction. Directly forecasting from the aggregate appears to unduly constrain the estimated parameters across countries, resulting in a poorer out-of-sample prediction.

\footnotetext{
${ }^{14}$ For brevity, we do not report all of these results in the paper but they are available from the authors upon request.
} 
However, in the case of inflation, the analysis is more ambiguous. For the EU12 and EU15 variables, pooled forecasts are more accurate at the 1-month horizon but less accurate at 3 and 12 months. However, differences between the two methods are smaller than for output growth. As expected, inflation rates have less diversity, given that a criterion for becoming a member of the euro area was that inflation rates could not vary by more than $2 \%$ of the lowest inflation country. Therefore, with less diversity in inflation rates, the gains in accuracy of one forecast method above another are likely to be small. However, for the EU25 country grouping, pooled forecasts are consistently more precise than those generated by the aggregate. Given the enlargement process now in place, this is a strong result for forecasters in the ECB charged with the development of appropriate economic policy across this diverse set of countries. Taking both forecast variables together for the enlarged group, our results support the use of pooling country-specific forecasts to predict the aggregate variable.

\section{CONCLUSIONS}

We compare forecasting models of economic growth and inflation in the context of an enlarged EU which now seeks to formulate economic policy to accommodate 25 countries. These countries differ greatly in terms of economic and financial development and this diversity makes the forecaster's problem more difficult. We focus on single linear equations which have been shown to perform relatively well in times of economic change. Specifically, we focus on a range of nested models using a simple AR as our benchmark. We augment this with a number of financial variables and test whether they add predictive content over and above that contained in the benchmark.

An important feature of our study is that we apply statistical tests to the evaluation of forecast accuracy and find that, even when models deliver a lower MSFE, this is often not statistically significant. Our main findings can be summarized in two parts. Firstly, at the country level, none of the financial variables systematically outperform the benchmark. Admittedly, most variables manage to improve forecast precision for some country and at some horizon, but it is not possible to identify a reliable pattern that would allow a forecaster to confidently select a particular variable to predict output growth or inflation at the country level. Furthermore, there is little statistical evidence that the general model should be preferred to its more restricted alternatives.

Secondly, at the aggregate level, our results are more promising. For output growth, using pooled forecasts from individual country models deliver lower MSFEs than those generated directly from the aggregate variable. This result always holds for GDP-weighted pooled forecasts. This improvement comes from allowing the estimated equation parameters to differ across countries and thus accounting for country heterogeneity. This is important for ECB forecasters to bear in mind when constructing forecasts. For inflation, a similar conclusion is reached when dealing with the EU25 country grouping - again an average of country forecasts weighted by GDP shares consistently outperforms forecasts of the aggregated variable for both the benchmark and best-performing models. For the EU12 and EU15 variable forecasts, our findings are less definite. However, at shorter horizons, the differences are small. In general, our results prescribe that forecasts should be formed by constructing GDP weighted averages of country forecasts, especially for the most diverse EU25 group. Furthermore, our chosen financial variables also deliver more consistent performance over differing forecast horizons. In particular, we find that adding stock market returns to the benchmark model improves the forecast accuracy of output growth for the EU12 and EU15 aggregates at all time horizons. Likewise for inflation, the economic growth variables always add a statistically significant improvement to the precision of the forecast in the EU25. 


\section{APPENDIX}

\section{Growth: industrial production index}

IFS: Austria, Cyprus, Czech Republic, Hungary, Belgium, Finland, Slovakia and Slovenia. Ecowin: Latvia. Datastream: Lithuania. Eurostat: The remaining. Fred Database: USA. Malta growth was proxied by interpolated GDP growth (source: IFS).

\section{Inflation: harmonized consumer price index}

IFS: The majority of countries with the exception of Cyprus, Portugal (Datastream), Ireland (Ecowin) and USA (FRED Database).

\section{Exchange rates: vis-à-vis the US dollar}

Source: IFS (Lithuania was not included due to a fixed exchange rate regime).

\section{Monetary aggregates}

Eurostat (M3 money supply): Austria, Belgium, Finland, France, Germany, Greece, Ireland, Luxembourg, Malta, Netherlands, Portugal, Spain; Central bank (M2 money supply): Cyprus, Czech Republic, Hungary, Lithuania, Poland, Slovakia, Slovenia; Ecowin (M2 money supply): Estonia, Latvia; Ecowin (M3 money supply): Denmark, Italy, Sweden, UK.

\section{Stock market: aggregate stock market index}

Datastream: the majority of countries (series TOTMKxx) with the exception of Estonia, Latvia, Lithuania, Malta, Slovakia and Slovenia (Ecowin).

\section{Interest rates: short term}

Ecowin (3-month T-bill): Austria, Belgium, Denmark, Finland, France, Germany, Italy, Netherlands, Spain, Sweden, UK; Ecowin (3-month deposit rate): Estonia, Greece, Ireland, Latvia, Portugal; Central bank(T-bill): Cyprus; IFS (T-bill): Czech Republic, Hungary, Malta; IFS (Money-market rate): Lithuania; IFS (deposit rate): Poland, Slovakia and Slovenia; Ecowin (Government benchmark bond): Luxembourg.

\section{ACKNOWLEDGEMENTS}

We are grateful to W. T. Gavin, A. Xepapadeas and participants at the 11th International Conference on Macroeconomic Analysis and International Finance and the 5th INFINITI Conference on International Finance for helpful comments and suggestions. The usual disclaimer applies.

\section{REFERENCES}

Ang A, Bekaert G, Wei M. 2007. Do macro variables, asset markets or surveys forecast inflation better? Journal of Monetary Economics 54: 1163-1212.

Banerjee A, Marcellino M. 2006. Are there any reliable leading indicators for US inflation and GDP growth? International Journal of Forecasting 22: 137-151. 
Barro R. 1990. The stock market and investment. Review of Financial Studies 3: 115-131.

Bernanke BS, Blinder AS. 1992. The federal funds rate and the channels of monetary transmission. American Economic Review 82: 901-921.

Davis EP, Fagan G. 1997. Are financial spreads useful indicators of future inflation and output growth in EU countries? Journal of Applied Econometrics 12: 701-714.

Diebold F, Mariano RS. 1995. Comparing predictive accuracy. Journal of Business and Economic Statistics 13: 253-263.

Estrella A, Mishkin F. 1998. Predicting US recessions: financial variables as leading indicators. Review of Economics and Statistics 80: 45-61.

Fama E. 1990. Stock returns, expected returns and real activity. Journal of Finance 45: 1089-1108.

Favero CA, Marcellino M. 2005. Modelling and forecasting fiscal variables for the Euro area. Oxford Bulletin of Economics and Statistics 67: 755-783.

Goodhart C, Hoffman B. 2000a. Do asset prices help to predict consumer price inflation. Manchester School 68: $122-140$

Goodhart C, Hoffman B. 2000b. Financial variables and the conduct of monetary policy. working Paper 112, Sveriges Riksbank.

Harvey CR. 1988. The real term structure and consumption growth. Journal of Financial Economics 22: 305-333.

Hassapis C. 2003. Financial variables and real activity in Canada. Canadian Journal of Economics 36: 421-442.

Hassapis C, Kalyvitis S. 2002. Investigating the links between growth and stock price changes with empirical evidence from the G7 economies. Quarterly Review of Economics and Finance 42: 543-575.

Haubrich JG, Dombrosky AM. 1996. Predicting real growth using the yield curve. Federal Reserve Bank of Cleveland Economic Review 32: 26-34.

Hendry DF, Clements MP. 2004. Pooling of forecasts. Econometrics Journal 7: 1-31.

Ing C-K. 2003. Multistep prediction in autoregressive processes. Econometric Theory 19: 254-279.

Lee BS. 1992. Causal relationships among stock returns, interest rates, real activity and inflation. Journal of Finance 47: 1591-1603.

Lutkepohl H. 1987. Forecasting Aggregated Vector ARMA processes. Springer: Berlin.

Marcellino M, Stock J, Watson M. 2003. Macroeconomic forecasting in the Euro area: country specific versus area-wide information. European Economic Review 47: 1-18.

McCracken MW. 2007. Asymptotics for out-of-sample tests of Granger causality. Journal of Econometrics 140: 719-752.

Panopoulou E, Pittis N, Kalyvitis S. 2005. Looking far in the past: re-visiting the growth-returns nexus with nonparametric tests. Available: http://www.aueb.gr/users/kalyvitis/[28 September 2008].

Stock JH, Watson MW. 1989. New indexes of coincident and leading economic Indicators. In NBER Macroeconomic Annual, Blanchard OJ, Fischer S (eds). MIT Press: Cambridge, MA; 351-394.

Stock JH, Watson MW. 1999. Business cycle fluctuations in US macroeconomic time series. In Handbook of Macroeconomics, Vol. 1A, Taylor JB, Woodford M (eds). North-Holland: Amsterdam; 3-64.

Stock JH, Watson MW. 2003. Forecasting output and inflation: the role of asset prices. Journal of Economic Literature 41: 788-829.

Stock JH, Watson MW. 2004. Combination forecasts of output growth in a seven-country data set. Journal of Forecasting 23: 405-430.

Swanson NR, White H. 1997. A model selection approach to real time macroeconomic forecasting using linear models and artificial neural networks. Review of Economic Statistics 13: 540-550. 